Article

\title{
Decoupling Adaptive Smith Prediction Model of Flatness Closed-Loop Control and Its Application
}

\author{
Mingming Song ${ }^{1}$, Hongmin Liu ${ }^{1,2}, *$, Yanghuan $\mathrm{Xu}^{1}{ }^{1}$, Dongcheng Wang ${ }^{1,2}$ and Yangyang Huang ${ }^{1}$ \\ 1 National Engineering Research Center for Equipment and Technology of Cold Rolling Strip, \\ Yanshan University, Qinhuangdao 066004, China; smm19891123@126.com (M.S.); \\ xuyanghuan011@126.com (Y.X.); wdc-731@163.com (D.W.); hyy_405@163.com (Y.H.) \\ 2 State Key Laboratory of Metastable Materials Science and Technology, Yanshan University, \\ Qinhuangdao 066004, China \\ * Correspondence: liuhmin@ysu.edu.cn
}

Received: 6 July 2020; Accepted: 22 July 2020; Published: 26 July 2020

check for updates

\begin{abstract}
Flatness control system is characterized by multi-parameters, strong coupling, pure time delay, which complicate the establishment of an accurate mathematical model. Therefore, a control scheme that combines dynamic decoupling, PI (Proportion and Integral) control and adaptive Smith predictive compensation is proposed. To this end, a dynamic matrix is used to decouple the control system. A multivariable coupled pure time-delay system is transformed into several independent generalized single-loop pure time-delay systems. Then, a PI-adaptive Smith predictive controller is constructed for the decoupled generalized single-loop pure time-delay system. Simulations show that the scheme has a simple and feasible structure, and good control performance. When the mathematical model of the control system is inaccurate, the control performance of adaptive Smith control method is evidently better than that of the ordinary Smith control method. The model is successfully applied to the cold rolling production site through LabVIEW, and the control accuracy is within 5I. This study reveals a new solution to the problem of coupled pure time-delay in flatness control system.
\end{abstract}

Keywords: cold rolling; flatness control; dynamic decoupling; pure time-delay; adaptive Smith

\section{Introduction}

Cold rolling strip is characterized by high speed (rolling speed), heavy load, large width-thickness ratio, multi-parameters etc. It requires high dimensional accuracy (micron level) and short measurement and control period (millisecond level). In recent years, with the development of science and technology, some industries, such as automobiles, household appliances, precision instruments, aerospace equipment, etc., have higher and higher requirements on the quality of strip flatness. It is urgent to eliminate the strip flatness defects as much as possible [1]. The flatness control technology of cold rolling strip has a complex principle, numerous influencing factors and a strong technical comprehensiveness. It is involved in different fields, such as mechanical, material, instrument, control, electronics, information and computer, among others. It has also been a trending but complex research topic locally and abroad for decades [2-8]. Firstly, available flatness actuators, and the components that characterize flatness, are numerous; moreover, each adjustment actuator affects each flatness component, thereby making flatness control a multi-input and multi-output (MIMO) coupling control system [9]. Secondly, there is a certain distance between the position of the shapemeter and the roll gap. A period is spent for the shapemeter to measure the flatness after rolling, thereby making flatness control a typical pure time-delay control system. Thirdly, the open-loop gain matrix (influence matrix) calculated by the flatness control deformation mechanism model according to the set parameters 
deviates from the actual open-loop gain matrix because the actual rolling parameters fluctuate at any time [10]. Therefore, an accurate flatness control model is difficult to obtain.

In conclusion, the flatness control system is multi-parameter and has strong coupling and pure time-delay. As a result, the control model is difficult to establish timely and accurately, thereby increasing the instability and design difficulty of the system. For this reason, Wang et al., [11] proposed the Smith predictive control method, Ma [12], proposed the Dahlin-Smith predictive control algorithm and Liu et al., [13] proposed the dynamic variable gain control algorithm. Their studies were all based on the multi-point flatness control method, which has a large calculation amount and low calculation efficiency [14]. Furthermore, the control effect is poor when the model mismatches. Li Bo et al. [15] proposed a predictive control method, and Zhang et al., [16] proposed the Smith predictive control method. Their studies were based on the component flatness control method, which has a small calculation amount and high calculation efficiency. However, they all believed that an actuator only affects one flatness component, thereby neglecting the coupling effect of each flatness control loop. Moreover, the control effect is poor when the model is mismatched.

Following the above analysis, this study presents a decoupling adaptive Smith predictive control algorithm based on component flatness control. This algorithm handles the coupling and pure time-delay of the loop respectively, and simplifies the design of the control system. Initially, a multivariable coupled pure time-delay system is decoupled into several generalized single variable pure time-delay systems by using the dynamic decoupling matrix. Then, for the generalized single-loop pure time-delay system, an adaptive Smith predictor is designed to solve the problem of pure time-delay, and improve the control performance when the model is mismatched [17]. In this control system, the transverse multi-point flatness is recognized as several characteristic components by pattern recognition [18], and the overall flatness is controlled by controlling the characteristic components. Compared with the multi-point flatness control system, the calculation amount is greatly reduced. Based on the theory of MIMO, the essence of the flatness control system is revealed. The whole process decoupling of the control system is realized by using the diagonal matrix decoupling method. The overshoot and adjustment time of the system are reduced by using the adaptive Smith method. Compared with other components flatness control system, the control process is more stable and faster.

\section{Flatness Decoupling Control System}

\subsection{Principle of Flatness Control}

The position of intermediate roll shifting (IRS) of a 1420mm UCM (universal crown control mill) six-high cold rolling mill will be set and kept unchanged according to the rolling process before rolling, which is called set value control. Therefore, there are only three kinds of on-line adjustment means, roll tilting (RT), work roll bending (WRB) and intermediate roll bending (IRB). Following rolling deformation theory and the engineering practice, RT controls primary flatness mainly, whereas WRB and IRB control quadratic and quartic flatness mainly. Figure 1 shows the principle of flatness control, in which, $\sigma=\left\{\sigma_{1}, \sigma_{2}, \ldots, \sigma_{\mathrm{m}}\right\}$ is a vector of the flatness of each section along the strip width direction measured by the shapemeter, and $\mathrm{m}$ is the number of measurement sections, where $\sigma_{\mathrm{m}}$ is the measured flatness value of the $\mathrm{m}$-th measurement section. After flatness pattern recognition the measured flatness component coefficient vector $A=\left\{a_{1}, a_{2}, a_{4}\right\}$ can be obtained, where $a_{1}, a_{2}$ and $a_{4}$, represent the measured primary, quadratic and quartic flatness component coefficients, respectively. $A^{T}=\left\{a_{1}^{T}, a_{2}^{T}, a_{4}^{T}\right\}$ is the target flatness component coefficient vector, $a_{1}^{T}, a_{2}^{T}$ and $a_{4}^{T}$ represent the primary, quadratic and quartic target flatness component coefficient respectively. $E=A^{T}-A=\left\{e_{1}, e_{2}, e_{4}\right\}$ is the vector of flatness deviation component coefficient, where $e_{1}, e_{2}$ and $e_{4}$ represent the primary, quadratic and quartic flatness deviation coefficient components respectively. $\Delta U=\left\{\Delta u_{1}, \Delta u_{2}, \Delta u_{4}\right\}$ is the regulator vector of a given flatness adjustment parameter, where $\Delta u_{1}, \Delta u_{2}$ and $\Delta u_{4}$ are the given RT displacement adjustment, WRB and IRB force adjustment respectively. $\Delta U^{*}=\left\{\Delta u_{1}^{*}, \Delta u_{2}^{*}, \Delta u_{4}^{*}\right\}$ is the actual output flatness adjustment parameter adjustment vector, $\Delta u_{1}^{*}, \Delta u_{2}^{*}$ and $\Delta u_{4}^{*}$ are the actual adjustment of RT 
displacement adjustment, WRB and IRB force adjustment respectively. In each control cycle, the flatness control system makes the target flatness $A^{T}$ minus the measured flatness $A$ initially obtain the flatness deviation $E$. Then, it calculates the adjustment amount $\Delta U$ according to the flatness deviation $E$ and sends it to the hydraulic system. Lastly, the hydraulic system of each actuator outputs the actual adjustment amount $\Delta U^{*}$ to the roll system according to its dynamic response. Meanwhile, the flatness of the roll gap is changed by changing its shape. The flatness after rolling reaches the shapemeter after $\tau=l / v$ time in which $l(\mathrm{~m})$ is the distance between the roll gap and the shapemeter, and $v(\mathrm{~m} / \mathrm{s})$ is the rolling speed.

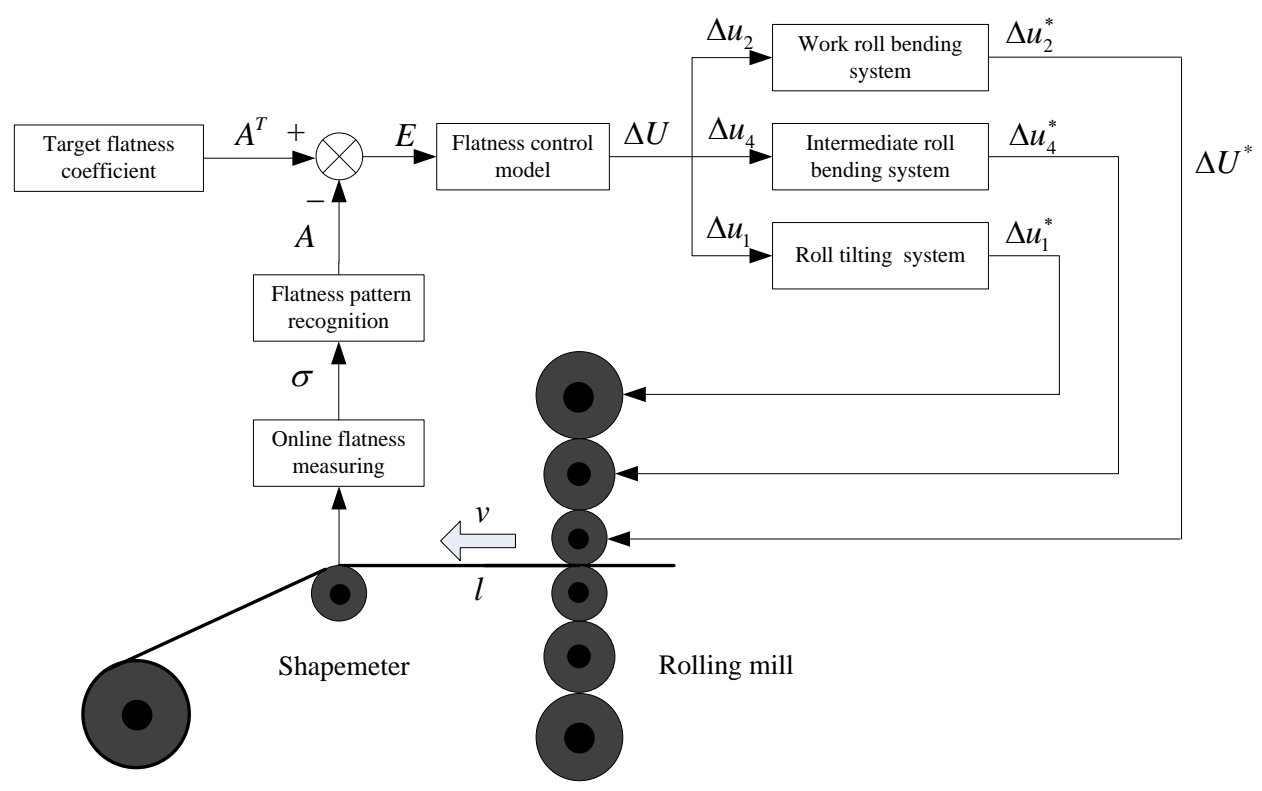

Figure 1. Flatness control principle.

\subsection{Flatness Open-Loop Control Model}

The flatness control system of the $1420 \mathrm{~mm}$ UCM six-roll cold rolling mill shown in Figure 1 is a three-input-three-output MIMO coupling pure time-delay control system. In accordance with the flatness control principle in Figure 1, the flatness open-loop control system which is shown in Figure 2 is constructed; in such system, $G_{T M}(s)=G_{T}(s) G_{M}(s), G_{W M}(s)=G_{W}(s) G_{M}(s), G_{I M}(s)=G_{I}(s) G_{M}(s)$, where $G_{T}(s), G_{W}(s), G_{I}(s)$ and $G_{M}(s)$ are the transfer functions of RT, WRB, IRB and shapemeter, respectively. Here, $\Delta a_{1}, \Delta a_{2}$ and $\Delta a_{4}$ are the changes of the flatness coefficients of primary, quadratic and quartic flatness, respectively; meanwhile, $a_{1}^{0}, a_{2}^{0}$ and $a_{4}^{0}$ are the initial measured (last time) primary, quadratic and quartic flatness coefficients respectively. Element $c_{i j}$ is the open-loop gain of the flatness control system, whose physical meaning is the influence coefficient of the $j$-th adjustment means on the $i$-th flatness component coefficient. The open-loop gain matrix of the flatness control is constituted by flatness component coefficient, which is called the flatness adjustment influence matrix C [19].

$$
C=\left[\begin{array}{lll}
c_{11} & c_{12} & c_{13} \\
c_{21} & c_{22} & c_{23} \\
c_{41} & c_{42} & c_{43}
\end{array}\right] c_{i j}=\Delta a_{i} / \Delta u_{j}^{*}
$$




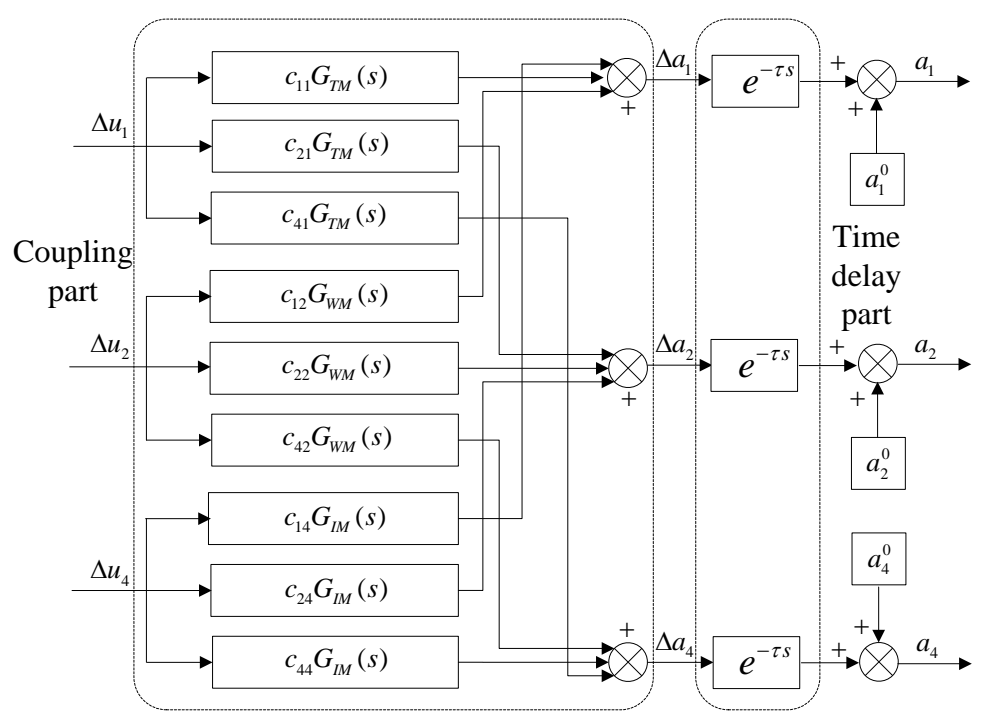

Figure 2. Open-loop control system.

Equation (1), $\Delta a_{\mathrm{i}}$ is the change of the $i$-th flatness component and $\Delta u_{j}^{*}$ is the change of the $j$-th actuator. The transfer functions of hydraulic system of RT, WRB and IRB of the cold rolling mill and the shapemeter are shown in Equation (2), which can be approximated to a first-order model [20].

$$
G_{T}(s)=\frac{1}{T_{T} s+1}, G_{W}(s)=\frac{1}{T_{W} s+1}, G_{I}(s)=\frac{1}{T_{I} s+1}, G_{M}(s)=\frac{1}{T_{M^{s}}+1}
$$

Equation (2), $s$ is the variable after Laplace transformation (differential operator). $T_{T}, T_{W}, T_{I}$ and $T_{M}$ are the time constants of the RT, WRB, and IRB system and the shapemeter, respectively. After the transfer function of each link and the influence matrix of flatness adjustment are known, the transfer function matrix $\mathrm{G}(\mathrm{s})$ of flatness control is obtained as follows. Equation (3), $e^{-\tau s}$ indicates that the system has a time-delay of $\tau$ seconds.

$$
G(s)=\left[\begin{array}{lll}
c_{11} G_{T M} e^{-\tau s} & c_{12} G_{W M} e^{-\tau s} & c_{14} G_{I M} e^{-\tau s} \\
c_{21} G_{T M} e^{-\tau s} & c_{22} G_{W M} e^{-\tau s} & c_{24} G_{I M} e^{-\tau s} \\
c_{41} G_{T M} e^{-\tau s} & c_{42} G_{W M} e^{-\tau s} & c_{44} G_{I M} e^{-\tau s}
\end{array}\right]
$$

\subsection{Flatness Closed-Loop Decoupling Control Model}

The pure time-delay time of each flatness component control loop is the same. Hence, the coupling and the time-delay parts of the strip control system can be dealt, respectively. For the coupling part in Figure 2, the coupling system can be transformed into three generalized single-loop control systems by decoupling. In this study, the closed-loop control strategy of independent control primary flatness, decoupling control quadratic and quartic flatness proposed by the author in another study is adopted [21]. The slight influence of RT on quadratic and quartic flatness and the slight influence of WRB and IRB on primary flatness are ignored. Figure 3 shows the flatness closed-loop decoupling control system. The dotted lines in Figure 3 is a dynamic decoupling model. $G_{D 22}(s), G_{D 42}(s)$, $G_{D 24}(s)$ and $G_{D 44}(s)$ are the four elements of the dynamic decoupling matrix $G_{D}(s) . G_{1}(s), G_{2}(s)$ and $G_{4}(s)$ are primary, quadratic and quartic flatness PI controllers, respectively. $E^{*}=\left\{e_{1}^{*}, e_{2}^{*}, e_{4}^{*}\right\}$ is the coefficient vector of flatness deviation component output by PI controllers, in which $e_{1}^{*}, e_{2}^{*}$ and $e_{4}^{*}$ are the primary, quadratic and quartic flatness deviation components output by PI controllers, respectively. In accordance with diagonal matrix decoupling theory [22], the control system will be decoupled 
completely when the dynamic decoupling matrix satisfies Equation (4), and the Equation (5) is obtained, which is called dynamic decoupling matrix.

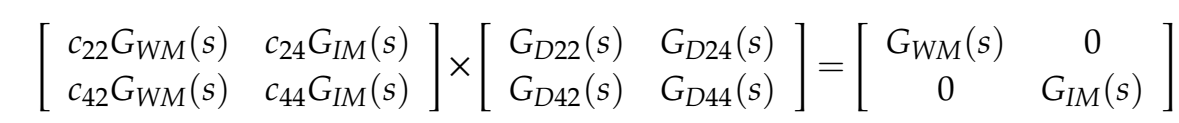

$$
\begin{aligned}
& G_{D}(s)=\frac{1}{c_{22} c_{44}-c_{24} c_{42}}\left[\begin{array}{cc}
c_{44} & -c_{24} G_{I M}(s) / G_{W M}(s) \\
-c_{42} G_{W M}(s) / G_{I M}(s) & c_{22}
\end{array}\right]
\end{aligned}
$$

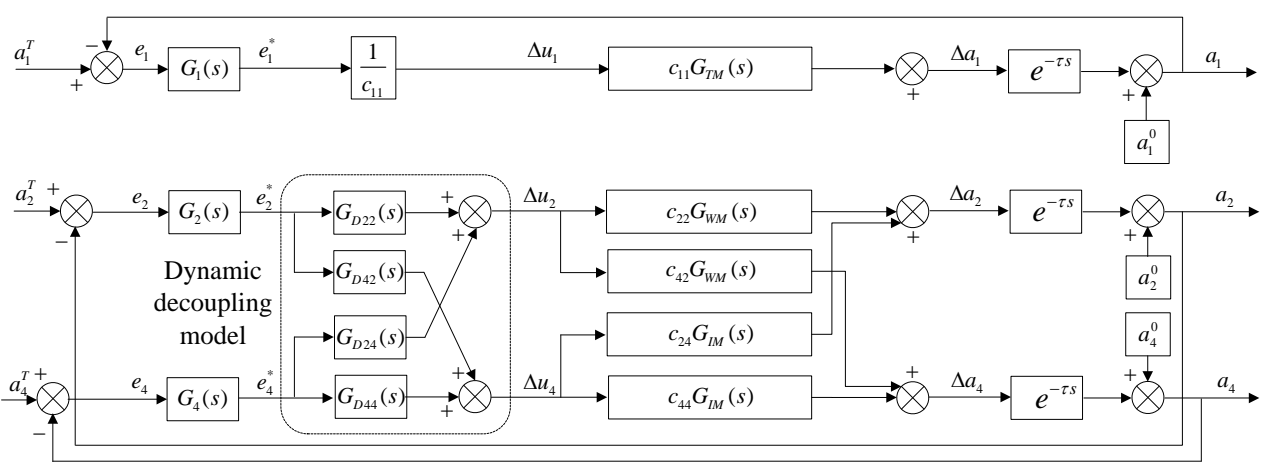

Figure 3. Closed-loop decoupling control system.

\section{Flatness Decoupling Adaptive Smith Predictive Control}

\subsection{Primary Flatness Adaptive Smith Predictive Control}

Decoupling control solves the coupling problem of the flatness control system, but does not solve the pure time-delay problem. The decoupled flatness control system can be regarded as three independent loops, and the predictive controller can be designed by solving single-loop pure time-delay; in this manner, the controller design is simplified and the practicability of the controller is enhanced $[23,24]$. Smith predictive control is an effective solution to the problem of single-loop pure time-delay. However, it depends on an accurate control object model to obtain good control effect $[25,26]$. However, in the general industrial process, the control object often contains uncertainties. As a result, the predictive model is difficult to match with the control object completely. Therefore, the desired control effect is difficult to achieve when the traditional Smith predictive control algorithm used [27]. In view of this deficiency of Smith predictive control, an adaptive Smith predictive control algorithm is proposed. By adjusting the parameters of the cascade controller adaptively, the output of the controller can be adjusted in real time to reduce the overshoot and adjustment time caused by model mismatch and enhance the stability of the system. Each control loop can be described by a first-order plus pure time-delay model because $T_{M}$ is more than one order of magnitude smaller than $T_{T}, T_{W}$ and $T_{I}$. In other words, $G_{T M}(s) \approx G_{T}(s), G_{W M}(s) \approx G_{W}(s)$ and $G_{I M}(s) \approx G_{I}(s)$ as shown in Figure 3. By taking the primary flatness control loop as an example, and ignoring the slight influence of WRB and IRB on primary flatness, the cascade controller adopts proportional control $K_{1}(t)$ to obtain a PI-adaptive Smith predictive control system of primary flatness as shown in Figure 4 , where, $K_{1}^{*}$, $G_{T M}^{*}(s)$ and $\tau^{*}$ are the open-loop gain coefficient, dynamic response and delay time of the primary flatness Smith predictor, respectively. $\Delta a_{1}^{*}$ is the primary flatness change output of the Smith predictor, and $e_{y 1}$ is the deviation between the actual and predicted outputs of the primary flatness control loop. 


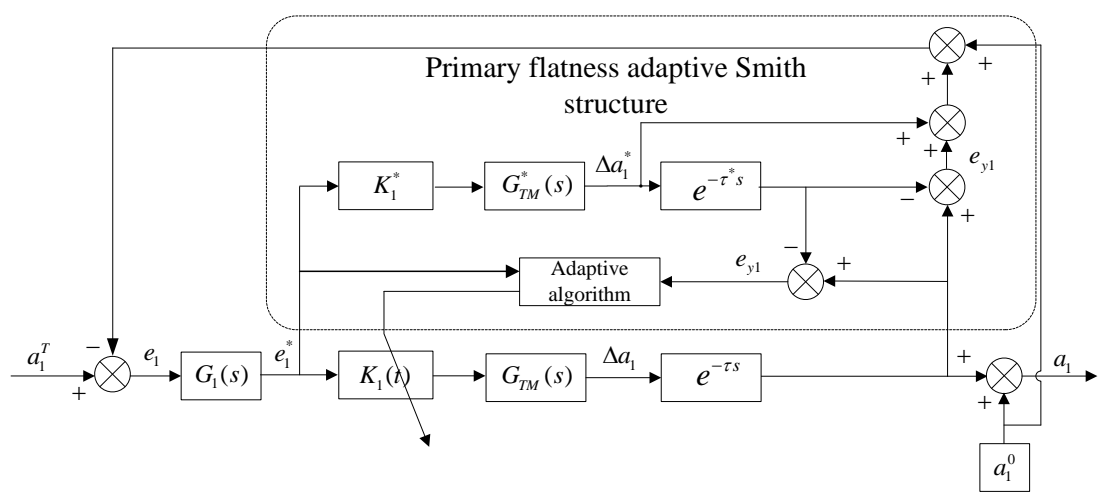

Figure 4. Flatness adaptive Smith control system.

Let $G_{T M}^{*}(s)=1 /\left(T_{T}^{*}+1\right)$, where $T_{T}^{*}$ is the time constant of Smith predictor, the following can be inferred from Figure 4. $\Delta a_{1}^{\prime}(\mathrm{t})$ and $\Delta a_{1}^{* \prime}(\mathrm{t})$ are the derivatives of $\Delta a_{1}(\mathrm{t})$ and $\Delta a_{1}^{*}(\mathrm{t})$ with respect to $\mathrm{t}$, respectively.

$$
\begin{gathered}
\Delta a_{1}^{\prime}(t)=-\frac{1}{T_{T}} \Delta a_{1}(t)+\frac{K_{1}(t)}{T_{T}} e_{1}^{*}(t) \\
\Delta a_{1}^{* \prime}(t)=-\frac{1}{T_{T}^{*}} \Delta a_{1}^{*}(t)+\frac{K_{1}^{*}}{T_{T}^{*}} e_{1}^{*}(t) \\
e_{y 1}(t)=\Delta a_{1}(t) e^{-\tau t}-\Delta a_{1}^{*}(t) e^{-\tau^{*} t}
\end{gathered}
$$

Given $G_{T M}^{*}(s)=1 /\left(T_{T}^{*}+1\right)$ and $T_{T}^{*}=T_{T}$, we can rewrite Equation (8) to Equation (9) because $e^{-\tau s}$ only shifts $\Delta a_{1}(t)$ and $\Delta a_{1}^{*}(t)$ on the time axis by a time $\tau$, which can be calculated, so the time-delay can be ignored [28]. Transforming Equation (8) to time domain and ignoring time-delay, it can be written as Equation (9).

$$
e_{y 1}(t)=\Delta a_{1}(t)-\Delta a_{1}^{*}(t)
$$

Taking the derivative of Equation (9), Equation (10) can be obtained.

$$
e_{y 1}^{\prime}(t)=-\frac{1}{T_{T}} e_{y 1}(t)+\frac{1}{T_{T}}\left[K_{1}(t)-K_{1}^{*}\right] e_{1}^{*}(t)
$$

Given $\Omega$ as a two-dimensional space containing variables $e_{y 1}(t)$ and $K_{1}(\mathrm{t})-K_{1}^{*}$, and $\varepsilon^{T}=\left\{e_{\mathrm{y} 1}(t)\right.$, $\left.\left(K_{1}(\mathrm{t})-K_{1}^{*}\right)\right\}$ as a vector of space $\Omega$, we choose Equation (11) as a Lyapunov-function [29].

$$
V(\varepsilon)=\frac{1}{2}\left\{T_{T} e_{y 1}^{2}(t)+\frac{1}{\lambda_{1}}\left[K_{1}(t)-K_{1}^{*}\right]^{2}\right\}
$$

Equation (11), $\lambda_{1}$ is a constant greater than zero, and $T_{T}>0$ holds for all $V(\varepsilon)>0$. Taking the derivative of Equation (11), Equation (12) can be obtained.

$$
V^{\prime}(\varepsilon)=T_{T} e_{y 1}(t) e_{y 1}^{\prime}(t)+\frac{1}{\lambda_{1}}\left[K_{1}(t)-K_{1}^{*}\right] K_{1}^{\prime}(t)
$$

By bring Equation (10) in Equation (12) and sorting out, Equation (13) can be obtained.

$$
V^{\prime}(\varepsilon)=-e_{y 1}^{2}(t)+\left[K_{1}(t)-K_{1}^{*}\right]\left[\frac{1}{\lambda_{1}} K^{\prime}(t)+e_{y 1}(t) e_{1}^{*}(t)\right]
$$

Given $K_{1}^{\prime}(t)=-\lambda_{1} e_{y 1}(t) e_{1}^{*}(t)$, Equation (14) can be obtained.

$$
K_{1}(t)=-\lambda_{1} \int e_{y 1}(t) e_{1}^{*}(t) d t+K_{1}(0)
$$




$$
V^{\prime}(\varepsilon)=-e_{y 1}^{2}(t) \leq 0
$$

Equation (14), $K_{1}(0)$ is the initial value of $K_{1}(t)$. Following Lyapunov's asymptotic stability theorem [30], Equation (10) is asymptotically stable, when $V^{\prime}(\varepsilon) \leq 0$. Equation (14) is the adaptive equation. To speed up the adjustment speed and improve the performance of the adaptive algorithm $K_{1}(\mathrm{t})$, an adaptive algorithm for the primary flatness is obtained as Equation (16) by adding $-\lambda_{1}^{*} e_{y 1}(t) e_{1}^{*}(t)$ ( $\lambda_{1}^{*}$ is a constant greater than zero) to the adaptive equation [31,32].

$$
K_{1}(t)=-\lambda_{1} \int e_{y 1}(t) e_{1}^{*}(t) d t-\lambda_{1}^{*} e_{y 1}(t) e_{1}^{*}(t)+K_{1}(0)
$$

\subsection{Quadratic and Quartic Flatness Decoupling Adaptive Smith Predictive Control}

The decoupled quadratic and quartic flatness control loops are two independent and uncoupled single-loops as shown in Figure 5. Each adaptive Smith predictive control system can be designed on the basis of single-loop. Ignoring the slight influence of RT on the quadratic and quartic flatness, and the cascade controllers adopt proportional control, the quadratic and quartic flatness decoupling PI-adaptive Smith predictive control system shown in Figure 6 is obtained. $K_{2}(t)$ and $K_{4}(t)$ are the quadratic and quartic flatness cascade proportional controllers, $K_{2}^{*}$ and $K_{4}^{*}$ are the open-loop gain coefficients of the quadratic and quartic flatness Smith predictors, $G_{W M}^{*}(s)$ and $G_{I M}^{*}(s)$ are the dynamic responses of the quadratic and quartic flatness Smith predictors, $\Delta a_{2}^{*}$ and $\Delta a_{4}^{*}$ are the quadratic and quartic flatness changes of the Smith predictors, and $e_{y 2}$ and $e_{y 4}$ are the deviation between the actual and predicted outputs of the quadratic and quartic flatness control loops respectively. Referring to the adaptive algorithm of primary flatness control, the adaptive algorithm of quadratic and quartic flatness control loops is obtained as shown in Equation (17) and Equation (18), respectively, in which $K_{2}(0)$ and $K_{4}(0)$ are the initial values of $K_{2}(t)$ and $K_{4}(t)$, respectively, and $\lambda_{2}, \lambda_{2}^{*}, \lambda_{4}$ and $\lambda_{4}^{*}$ are four constants greater than zero.

$$
\begin{aligned}
& K_{2}(t)=-\lambda_{2} \int e_{y 2}(t) e_{2}^{*}(t) d t-\lambda_{2}^{*} e_{y 2}(t) e_{2}^{*}(t)+K_{2}(0) \\
& K_{4}(t)=-\lambda_{4} \int e_{y 4}(t) e_{4}^{*}(t) d t-\lambda_{4}^{*} e_{y 4}(t) e_{4}^{*}(t)+K_{4}(0)
\end{aligned}
$$

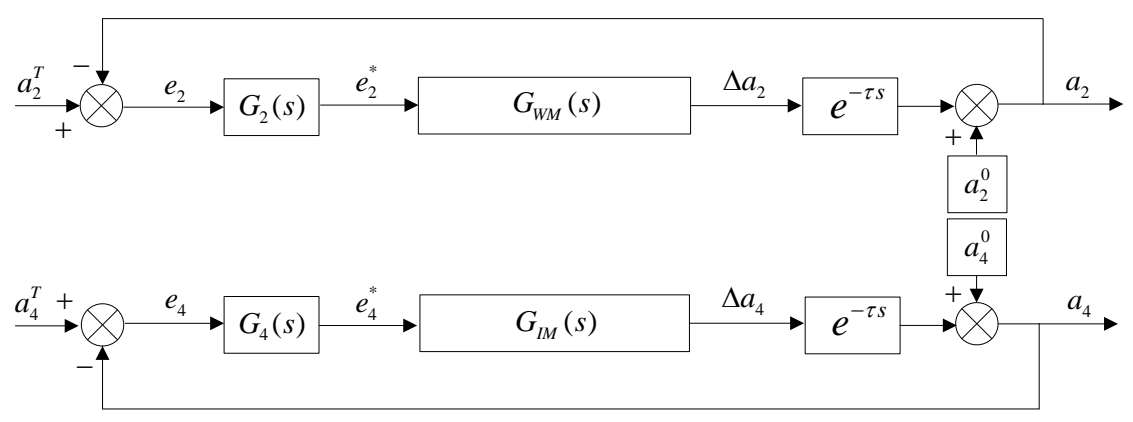

Figure 5. Generalization closed-loop control system of quadratic and quartic flatness. 


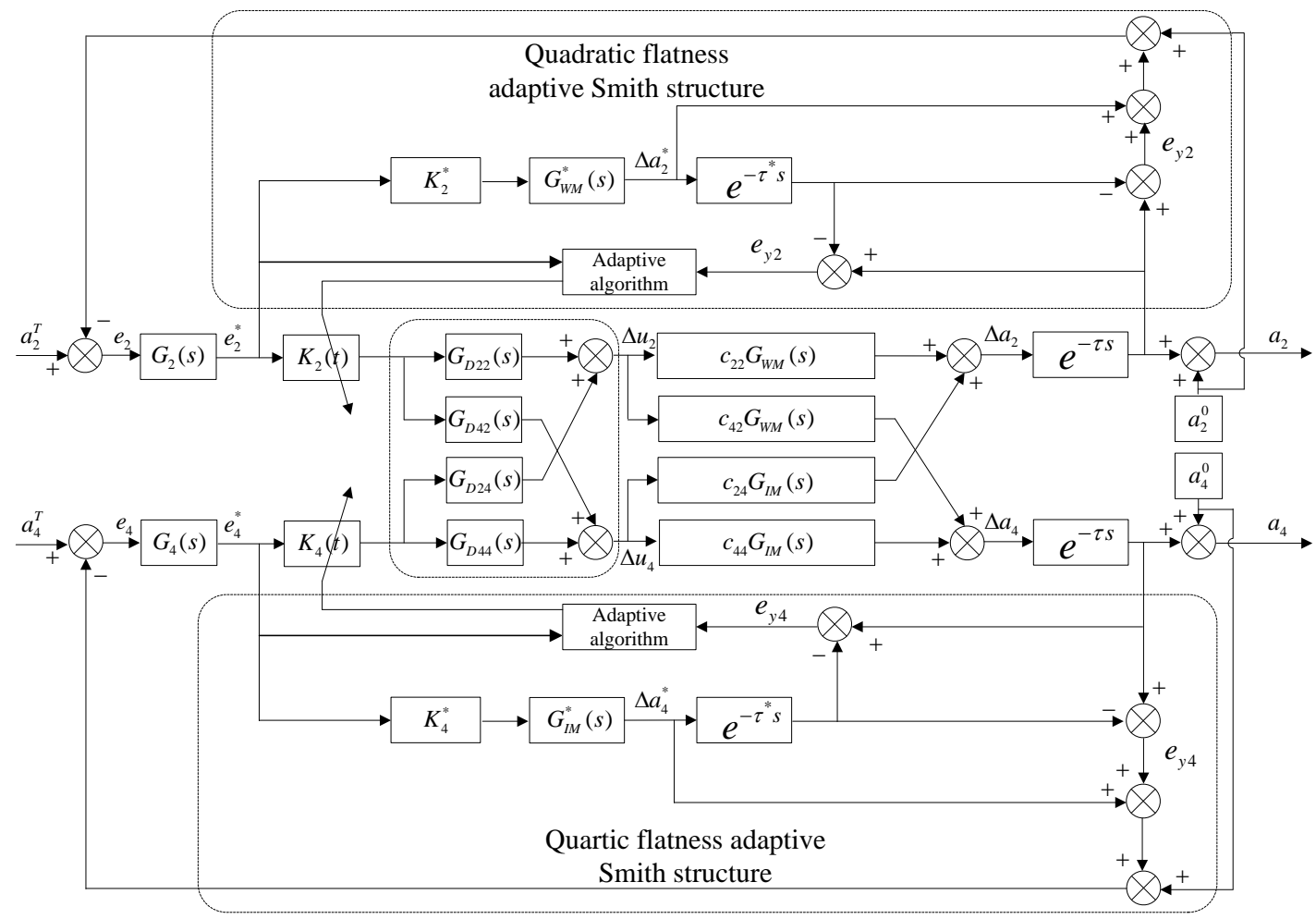

Figure 6. Quadratic and quartic flatness decoupling adaptive Smith control system.

\section{Simulation Comparison and Industrial Application}

\subsection{Simulation Comparison}

$T_{T}, T_{W}, T_{I}$ and $T_{M}$ are $0.05 \mathrm{~s}, 0.15 \mathrm{~s}, 0.25 \mathrm{~s}$ and $0.003 \mathrm{~s}$ of a $1420 \mathrm{~mm} \mathrm{UCM}$ six-roll cold rolling mill in a factory respectively. The normal rolling speed of the mill is between 5.0 and $13.3 \mathrm{~m} / \mathrm{s}$ (according to field rolling process), and the distance between the shapemeter and the roll gap is $2.0 \mathrm{~m}$ (field measurement). Therefore, the delay time is between 0.15 and $0.4 \mathrm{~s}$. The normal rolling speed of the strip with a width of $1230 \mathrm{~mm}$ is $10 \mathrm{~m} / \mathrm{s}$, i.e., delay time $\tau=0.2$ main rolling parameters and the flatness adjustment influence matrix (calculating matrix) calculated from the flatness control deformation mechanism model are shown in Table 1 [33]. The simulation systems of flatness control Simulink based on decoupling PI, decoupling PI-Smith and decoupling PI-adaptive Smith are built, respectively. Figure 5 shows that when the model matches perfectly, the open-loop gain coefficient of the generalized quadratic and quartic flatness control loops after decoupling is 1 . Hence, the initial value of the cascade controller of the quadratic and quartic flatness control loops and the open-loop gain of Smith predictor are all 1, i.e., $K_{2}(0)=1, K_{4}(0)=1, K_{2}^{*}=1$ and $K_{4}^{*}=1$, similarly $K_{1}(0)=1, K_{1}^{*}=1$. The parameters of each loop controller and the adaptive parameters are selected as shown in Table 2, in which $K_{P 1}, K_{I 1}, K_{P 2}$, $K_{I 2}, K_{P 4}$ and $K_{I 4}$ are the proportional and integral coefficients of the primary, quadratic and quartic flatness PI controllers, respectively. Taking the primary flatness control loop as an example, its PI controller equation is shown in Equation (19) [34].

$$
u_{t}=K_{p} e(t)+K_{I} \int e(t) d t
$$


Table 1. Main rolling parameters and calculating matrix.

\begin{tabular}{cccccccccc}
\hline $\begin{array}{c}\text { Strip } \\
\text { Width } \\
\text { /mm }\end{array}$ & $\begin{array}{c}\text { Rolling } \\
\text { Force } \\
/ \mathbf{1 0 k N}\end{array}$ & $\begin{array}{c}\text { Entry } \\
\text { Thickness } \\
/ \mathbf{m m}\end{array}$ & $\begin{array}{c}\text { Exit } \\
\text { Thickness } \\
/ \mathbf{m m}\end{array}$ & $\begin{array}{c}\text { Front } \\
\text { Force } \\
/ \mathbf{k N}\end{array}$ & $\begin{array}{c}\text { Post } \\
\text { Tension } \\
/ \mathbf{k N}\end{array}$ & $\begin{array}{c}\text { IRS } \\
/ \mathbf{m m}\end{array}$ & \multicolumn{3}{c}{$\begin{array}{c}\text { Calculating Matrix } \\
\mathbf{C}_{\mathbf{0}}\end{array}$} \\
\hline & & & & & & & -0.1829 & -0.0001 & 0.0002 \\
1230 & \multirow{2}{*}{864} & \multirow{2}{*}{1.04} & 0.79 & \multirow{2}{*}{107} & 80 & 115 & 0.0003 & -0.4084 & 0.0398 \\
& & & & & & & 0.0005 & 0.3079 & 0.0373 \\
\hline
\end{tabular}

Table 2. Main parameters of control system.

\begin{tabular}{cccccccccccc}
\hline$K_{P 1}$ & $K_{I 1}$ & $\lambda_{1}$ & $\lambda_{1}{ }^{*}$ & $K_{P 2}$ & $K_{I 2}$ & $\lambda_{2}$ & $\lambda_{2}{ }^{*}$ & $K_{P 4}$ & $K_{I 4}$ & $\lambda_{4}$ & $\lambda_{4}{ }^{*}$ \\
\hline 0.2 & 0.033 & 0.01 & 0.5 & 0.6 & 0.107 & 0.03 & 0.45 & 0.5 & 0.167 & 0.02 & 0.40 \\
\hline
\end{tabular}

In accordance with Equation (3), the transfer function matrix of the flatness control system is composed of three parts: influence matrix, dynamic response and time-delay. RT, WRB and IRB are the inner rings of flatness control. Their time constants, i.e., their dynamic response is determined. The delay time is $\tau=l / v$, where $l$ is fixed, and $v$ is measured in real time, so the delay time is determined. Therefore, the major factor affecting the transfer function matrix is the influence matrix. The influence matrix for control is usually calculated from the flatness control deformation mechanism model according to the set (specific) parameters, which is called calculating matrix. The actual rolling parameters fluctuate at any time, thereby result in a large deviation between the calculating matrix and the actual matrix, which can be calculated in accordance with the measured data of the rolling process and changes at any time. In summary, only the influence matrix is uncertain in the flatness control system model. Hence, in this study, only the matching and mismatching control performance comparison between the calculating matrix of the decoupling part and the actual matrix of the coupling part are discussed.

Assuming the initial flatness is $a_{1}^{0}=0, a_{2}^{0}=0$ and $a_{4}^{0}=0$, let $a_{1}^{T}=1, a_{2}^{T}=1$ and $a_{4}^{T}=1$, and the various situations of actual matrix and calculating matrix of rolling mill are simulated and compared. Three representative cases are presented in Table 3 and Figure 7. The interpretations of the curves in Figure 7 are shown in Table 4.

(1) From the comparison of Curves A and B, when the model matches perfectly, after adding Smith predictive control, the flatness components are not overshoot and the adjustment time is greatly shortened. Hence the control strategy of decoupling first and then Smith predictive control is effective.

(2) From the comparison of Curves C and D, when the absolute value of each element of the actual matrix is larger than that of the calculating matrix, PI-Smith predictive control produces large overshoots for each flatness component. After using PI-adaptive Smith predictive control, the overshoots of the primary and quadratic flatness are evidently reduced, the quartic flatness is no overshoot.

(3) From the comparison of Curves E and F, when the absolute value of each element in the actual matrix is smaller than that of the calculating matrix, and the adjustment time of each flatness component is long using PI-Smith predictive control, whereas that of PI-adaptive Smith predictive control is short.

In summary, the flatness control system using decoupled PI-adaptive Smith predictive control can greatly reduce overshoot caused by pure time-delay, shorten the adjustment time, and stabilize control process. Furthermore, the adaptive algorithm can improve the control quality of the system when the mathematical model is mismatched.

Table 3. Actual matrix.

\begin{tabular}{ccccccccc}
\hline \multicolumn{3}{c}{$\mathbf{C}_{\mathbf{0}}$} & \multicolumn{3}{c}{$\mathbf{C}_{\mathbf{1}}$} & \multicolumn{3}{c}{$\mathbf{C}_{\mathbf{2}}$} \\
\hline-0.1829 & -0.0001 & 0.0002 & -0.2560 & -0.0001 & 0.0003 & -0.1097 & -0.0001 & 0.0001 \\
0.0003 & -0.4084 & 0.0398 & 0.0004 & -0.5718 & 0.0557 & 0.0002 & -0.2450 & 0.0239 \\
0.0005 & 0.3079 & 0.0373 & 0.0007 & 0.4310 & 0.0522 & 0.0003 & 0.1847 & 0.0224 \\
\hline
\end{tabular}



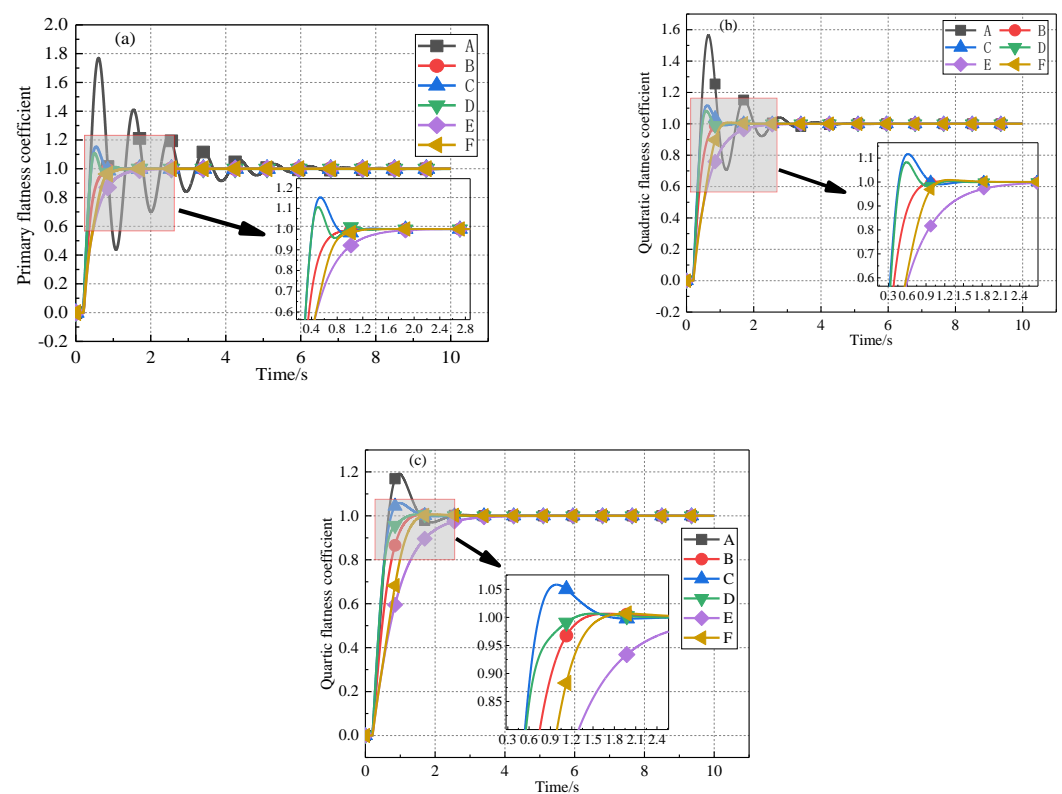

Figure 7. Contrast of flatness control change process.

Table 4. The meanings of the curves in Figure 7.

\begin{tabular}{ccccccc}
\hline Curve label & A & B & C & D & E & F \\
\hline Calculating matrix & $\mathrm{C}_{0}$ & $\mathrm{C}_{0}$ & $\mathrm{C}_{0}$ & $\mathrm{C}_{0}$ & $\mathrm{C}_{0}$ & $\mathrm{C}_{0}$ \\
\hline Actual matrix & $\mathrm{C}_{0}$ & $\mathrm{C}_{0}$ & $\mathrm{C}_{1}$ & $\mathrm{C}_{1}$ & $\mathrm{C}_{2}$ & $\mathrm{C}_{2}$ \\
\hline Control method & PI & PI-Smith & PI-Smith & PI-adaptive Smith & PI-Smith & PI-adaptive Smith \\
\hline
\end{tabular}

\subsection{Industrial Application}

The flatness control method proposed in this paper has been applied to a $1420 \mathrm{~mm}$ UCM six-high cold rolling mill. The control program is written with LabVIEW as the development environment, and its application process principle is shown in Figure 8. Ni OPC server is the bridge between LabVIEW and S7-400 to establish data connection, and plays the function of communication protocol conversion. The flatness control program first reads the parameters needed for flatness control in 57-400 through $\mathrm{Ni}$ OPC server, then reads the measured flatness of the flatness measuring host through the switch, calculates the flatness control amount and writes it into 57-400 through Ni OPC server, and finally, S7-400 gives the control adjustment amount to the corresponding actuator through its input and output (I/O) equipment. The variation range of $\mathrm{RT}$ is $-150 \mu \mathrm{m} \sim 150 \mu \mathrm{m}$, the variation range of WRB is $-200 \mathrm{kN} \sim 500 \mathrm{kN}$, the variation range of IRB is $0 \mathrm{kN} \sim 500 \mathrm{kN}$, and the variation range of IRS is $-200 \mathrm{~mm} \sim 200 \mathrm{~mm}$. Take the strip of Table 1 as an example, control period is $50 \mathrm{~ms}$, the target flatness $a_{1}^{T}=0, a_{2}^{T}=0$ and $a_{4}^{T}=0$, the initial RT is $0 \mu \mathrm{m}$, the initial WRB is $110 \mathrm{kN}$, the initial IRB is $120 \mathrm{kN}$, the actual change of flatness component coefficient, actuator adjustment amount, the overall flatness change process and the strip after rolling are shown in (a), (b), (c) and (d) of Figure 9, respectively. The flatness data is from the flatness control host (sampling period $50 \mathrm{~ms}$ ), and the actual change of actuator adjustment parameters is from the factory PDA (sampling period $1 \mathrm{~ms}$ ). 


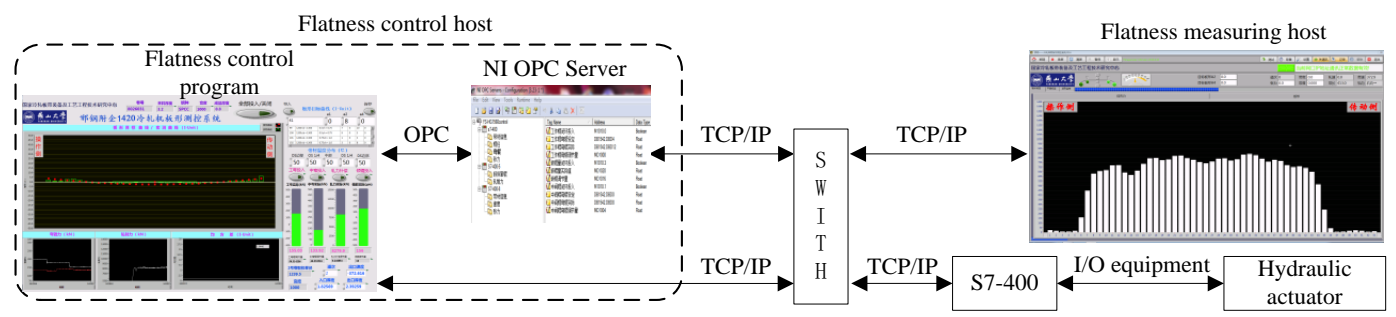

Figure 8. Principle of flatness control system.

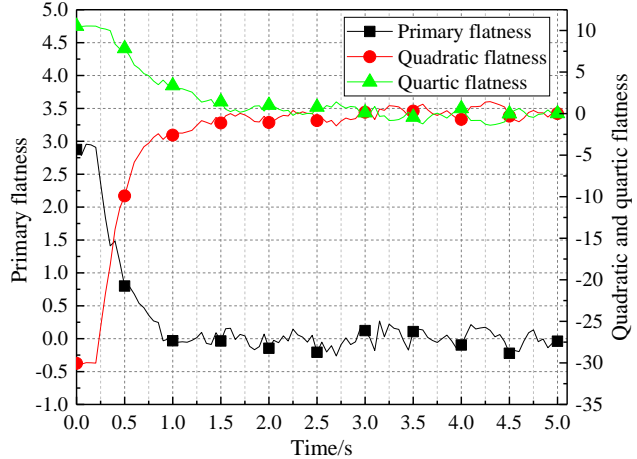

(a). Change process of flatness components.

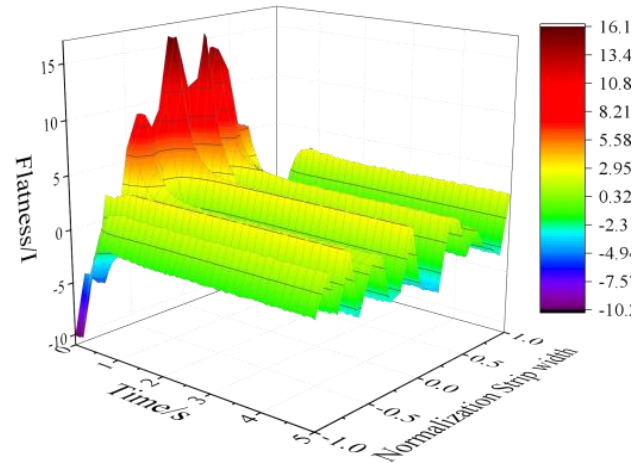

(c). Change process of the overall flatness.

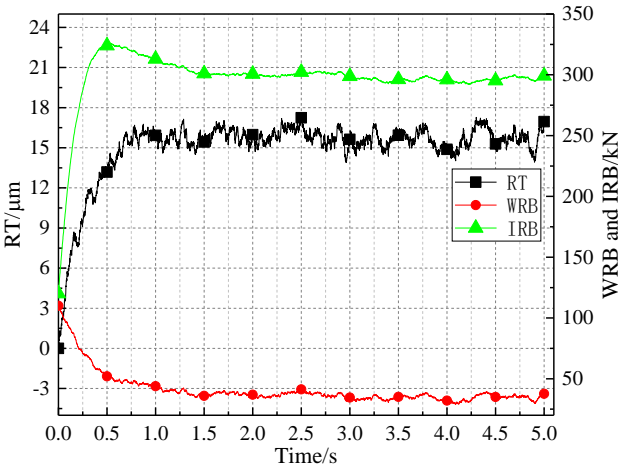

(b). Change process of actuator.

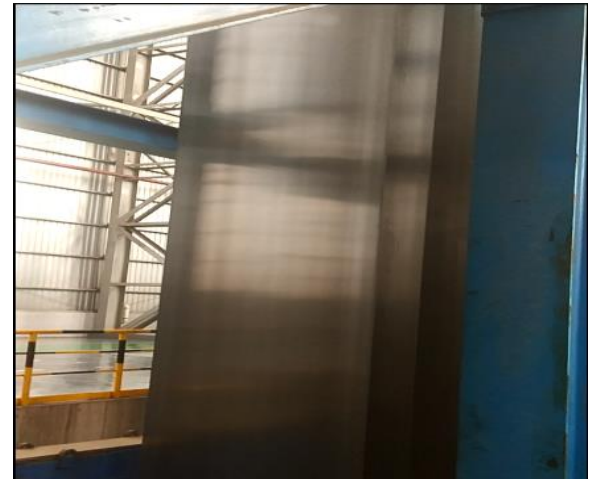

(d). The strips after rolling.

Figure 9. Change of control parameters in rolling process.

It can be seen that the controlled primary, quadratic and quartic flatness coefficients do not overshoot and reach the target value in about $1.5 \mathrm{~s}$. After control, the flatness of all transverse parts of the strip are within \pm 5 I. The remaining flatness defects are high-order, whose shape is wave, which indirectly shows that RT, WRB and IRB can't adjust the high-order flatness. In the process of adjustment, only the IRB produces overshoot of about $10 \%$, and the RT and WRB do not produce overshoot. The overall flatness of the strip changes rapidly and stably, and the surface shape of the strip is good after rolling without obvious deformation. After a long time of industrial application, different specifications of strip only have different response of the actuator and flatness during the adjustment process, the adjustment time is mostly within $2.5 \mathrm{~s}$, and the final flatness deviation is mostly within $\pm 5 \mathrm{I}$, which shows that the decoupling strategy and control method proposed in this paper are effective. With the setting value of IRS is appropriate, the integral flatness deviation of strip can be eliminated by adjusting the primary, quadratic and quartic flatness components through roll RT, WRB and IRB, and the local flatness deviation cannot be eliminated. 


\section{Discussion}

The flatness control system can control the overall flatness of strip by controlling the three characteristic components: primary, quadratic and quartic. Compared with the multi-point flatness control system in references $11-14$, the design is simple, the control variables are fewer, and the calculation amount is small. When the control system model is mismatched, the adjustment time of the system is shorter, the flatness control process is more stable and the actuator will not produce large overshoot. Compared with the predictive control algorithm in reference 15, the process coupling of the system is considered in this paper. The dynamic term is added to the decoupling process to realize the whole process decoupling of the control system. Moreover, the influence of various actuators means that the flatness components is considered, so the design of the control system is more perfect and the operation more stable. Compared with the traditional Smith predictive control algorithm in reference 16, the adaptive Smith algorithm proposed in this paper is more suitable for industrial field, the flatness control effect is better, the overshoot of actuator is smaller, and the adjustment time is shorter. Because the control system is specially designed for a $1420 \mathrm{~mm}$ UCM reversible cold rolling mill, it is not equipped with subsection cooling system, the system cannot be combined with subsection cooling, and the flatness control accuracy remains at $\pm 5 \mathrm{I}$, which cannot be further improved.

\section{Conclusions}

1. Through analysis the internal structure of the flatness control system, the control strategy of decoupling first and then compensating time-delay is proposed. A complex multi-loop coupled time-delay system is decoupled into simple multi-generalized single-loop time-delay systems. In this manner, the design difficulty of the control system is simplified.

2. To address solve the difficulty in accurately calculating the influence matrix of flatness adjustment and the poor effect of ordinary Smith predictive control method, this study proposes an adaptive Smith predictive control method. Adjusting the cascade controller coefficients in real time reduces the overshoot and adjustment time caused by model mismatch, improving the control quality.

3. The simulation results show that for the time-delay flatness control system Smith predictive control is effective when the model is matched. When the control model is mismatched, the adaptive Smith method is better than the ordinary Smith method.

4. The industrial application shows that the setting value of IRS is appropriate, the integral flatness deviation of strip can be controlled within $\pm 5 \mathrm{I}$ by adjusting the primary, quadratic and quartic flatness components, through roll RT, WRB and IRB, and the local flatness deviation cannot be eliminated.

Author Contributions: Conceptualization, M.S. and H.L.; methodology, M.S.; software, M.S.; validation, D.C.; formal analysis, Y.X.; investigation, M.S. and Y.H.; data curation, M.S.; writing-original draft, M.S.; project administration, H.L. All authors have read and agreed to the published version of the manuscript.

Funding: This work is supported by the National Science and Technology Planning Program (Grant No. 2011BAF15B00) and the Natural Science Foundation of Hebei Province (Grant No. E2016203482).

Conflicts of Interest: The authors declare no conflict of interest.

\section{References}

1. Yang, L.P.; Yu, H.X.; Zhang, Y.S.; Liu, H.M. Shape Detection and Control System of Cold Rolling Strip Based on the Virtual Instrument and Its Industrial Application. J. Mech. Eng. 2018, 54, 1-7. [CrossRef]

2. Li, X.; Luan, F.; Wu, Y.A. Comparative Assessment of Six Machine Learning Models for Prediction of Bending Force in Hot Strip Rolling Process. Metals 2020, 10, 685. [CrossRef]

3. Pin, G.; Francesconi, V.; Cuzzola, F.A.; Parisini, T. Adaptive task-space metal strip-flatness control in cold multi-roll mill stands. J. Process Control 2013, 23, 108-119. [CrossRef]

4. Jin, X.; Li, C.S.; Wang, Y.; Li, X.G.; Xiang, Y.; Gu, T. Investigation and Optimization of Load Distribution for Tandem Cold Steel Strip Rolling Process. Metals 2020, 10, 677. [CrossRef] 
5. Ogasahara, T.; Kitamura, T.; Aoe, S.I.; Tateno, J.I.; Asano, K. Dynamic Control of Flatness and Elongation of the Strip in a Skin Pass Mill. Tetsu Hagane 2019, 105, 512-521. [CrossRef]

6. Xie, H.B.; Li, L.J.; Liu, T.W.; Wang, E.R.; Liu, X.; Jiang, Z.Y. Three Dimensional Finite Element Simulation of Strip Shape and Flatness of High Strength Steel. Key Eng. Mater. 2019, 794, 232-245. [CrossRef]

7. Kozhevnikov, A.; Kozhevnikov, I.; Bolobanova, N. Dynamic model of cold strip rolling. Metalurgija 2018, 57, 99-102.

8. Ataka, M. Rolling Technology and Theory for the Last 100 Years: The Contribution of Theory to Innovation in Strip Rolling Technology. ISIJ Int. 2015, 55, 89-102. [CrossRef]

9. Liu, H.M.; He, H.T.; Shan, X.Y.; Jiang, G.B. Flatness Control Based on Dynamic Effective Matrix for Cold Strip Mills. J. Mech. Eng. 2009, 22, 41-75. [CrossRef]

10. Yang, L.P.; Yu, H.Y.; Wang, D.C.; Zhang, Z.; Jiang, Z.Y. Intelligent Shape Regulation Cooperative Model of Cold Rolling Strip and Its Application. Steel Res. Int. 2017, 88, 1600383. [CrossRef]

11. Wang, P.F.; Peng, Y.; Wang, D.C.; Sun, J.; Zhang, D.H.; Liu, H.M. Flatness Control Strategy Based on Delay Compensation for Cold Rolling Mill. Steel Res. Int. 2017, 88, 1600065.

12. Ma, L. Strategy of Flatness Control System Lag Compensation. Master's Thesis, Yanshan University, Qinhuangdao, China, 2014.

13. Liu, J.W.; Jiang, D.P.; Wu, H.L.; Chen, R.J. Algorithm Research and Application of dynamic variable gain controller based on flatness control in cold mill. In Proceedings of the 8th Youth Academic Annual Meeting of China Metal Society, Beijing, China, 23-25 October 2013; pp. 1-6.

14. Wang, P.F.; Zhang, Z.J.; Sun, J.; Zhang, D.H.; Liu, H.M.; Gao, X.L. Flatness control of cold rolled strip based on relay optimization. Ironmak. Steelmak. 2016, 45, 166-175. [CrossRef]

15. Li, B.; Zhang, Q.D. Study on Flatness Predictive Control for CVC-4th Cold Mill. Metall. Equip. 2008, 4, 9-12.

16. Zhang, R.C.; Zheng, X. Automatic flatness control strategy with a Smith predictor for steel strip rolling. In Proceedings of the 2011 International Conference on Photonics, 3D-Imaging, and Visualization, Guangzhou, China, 30-31 October 2011; pp. 1-6.

17. Xing, Z.W.; Ding, B.J. Intelligent Control Method for Aircraft Deicing Fluid temperature Based on a New Adaptive Smith Predictor. Adv. Mater. Res. 2012, 424, 936-940. [CrossRef]

18. Song, M.M.; Wang, D.C.; Zhang, S.; Xu, Y.H.; Liu, H.M. Flatness pattern recognition based on recurrent neural network. Iron Steel 2018, 53, 56-62.

19. Yang, L.P.; Zhang, Z.; Wang, D.C.; Li, R.M.; Yu, H.X.; Zhang, Y.S. Mechanism-intelligent coordination shape control model of cold strip. Iron Steel 2017, 52, 52-57.

20. Zhu, Y.; Tang, S.; Wang, C.; Jiang, W.; Zhao, J.; Li, G. Absolute stability condition derivation for position closed-loop system in hydraulic automatic gauge control. Processes 2019, 7, 766. [CrossRef]

21. Song, M.M.; Liu, H.M.; Wang, D.C.; Xu, Y.H. Decoupling Strategy and Dynamic Decoupling Model of Flatness Control in Cold Rolling Strip. ISIJ Int. 2020, 60, 286-296. [CrossRef]

22. Wang, J.; Yan, W.; Shao, H. Performance improvement of VAV air conditioning control system through diagonal matrix decoupling and Lonworks technology. Energy Build. 2005, 37, 911-919. [CrossRef]

23. Chou, J.H.; Chang, J.F.; Lin, D.B.; Wu, T.L. Dual-band WLAN MIMO antenna with a decoupling element for full-metallic bottom cover tablet computer applications. Microw. Opt. Technol. Lett. 2018, 60, 1245-1251. [CrossRef]

24. Xu, S.; Hashimoto, S.; Jiang, W. Pole-Zero Cancellation Method for Multi Input Multi Output (MIMO) Temperature Control in Heating Process System. Processes 2019, 7, 497. [CrossRef]

25. Kaya, I. Improving performance using cascade control and a Smith predictor. ISA Trans. 2001, 40, $223-234$. [CrossRef]

26. Wang, Y.Q.; Sun, F.; Liu, J.; Sun, M.H.; Xie, Y.H. Application of Smith Predictor Based on Single Neural Network in Cold Rolling Shape Control. Chin. J. Mech. Eng. 2009, 22, 282-286. [CrossRef]

27. Zhang, P.; Yuan, M.Z.; Wang, H. Self-adaptive Fuzzy-Smith Control for a Long Time-delay System. Comput. Simul. 2006, 23, 88-90.

28. Li, X.; Song, D.Q.; Yu, S.Y.; Gui, W.H. Feedback automatic gauge control system using model reference adaptive Smith predictor. Control Theory Appl. 2009, 26, 999-1003.

29. Gao, H.J.; Lam, J.; Chen, T.W.; Wang, C.H. Stability analysis of uncertain discrete-time systems with time-varying state delay: A parameter-dependent Lyapunov function approach. Asian J. Control 2010, 8, 433-440. [CrossRef] 
30. Zheng, D.Z. Liner System Theory; Tsinghua University Press: Beijing, China, 1990; pp. 213-242.

31. Zhou, Y.; Song, D.D.; Nie, P.P.; Hao, H.M. Application of model reference adaptive control-improved Smith-predictor in temperature control of fermentation. J. Hebei Univ. Tech. 2011, 40, 5-9.

32. Song, Y.X.; Zhu, X.F. An Improved Adaptive Smith Predictor System. Meas. Control Tech. 2002, 8, 37-40.

33. Wang, D.C.; Liu, H.M. A Model Coupling Method for Shape Prediction. J. Iron Steel Res. Int. 2012, $19,22-27$. [CrossRef]

34. Rodríguez-Miranda, E.; Beschi, M.; Guzmán, J.L.; Berenguel, M.; Visioli, A. Daytime/Nighttime Event-Based PI Control for the $\mathrm{pH}$ of a Microalgae Raceway Reactor. Processes 2019, 7, 247. [CrossRef] 